\title{
Correction to: Rescue of Methyl-CpG Binding Protein 2 Dysfunction-induced Defects in Newborn Neurons by Pentobarbital
}

\author{
Dongliang Ma ${ }^{1} \cdot$ Su-In Yoon ${ }^{1,2} \cdot$ Chih-Hao Yang ${ }^{3} \cdot$ Guillaume Marcy $^{1} \cdot \mathrm{Na}_{\text {Zhao }}{ }^{1,4} \cdot$ Wan-Ying Leong ${ }^{1}$. \\ Vinu Ganapathy ${ }^{1}$ • Ju Han ${ }^{1}$. Antonius M. J. Van Dongen ${ }^{1,5}$ • Kuei-Sen Hsu ${ }^{6}$ Guo-Li Ming ${ }^{7}$. \\ George J. Augustine ${ }^{1,2,5,8,9}$ • Eyleen L. K. Goh ${ }^{1,2,5,10}$
}

Published online: 30 April 2019

(C) The American Society for Experimental NeuroTherapeutics, Inc. 2019

\section{Correction to: Neurotherapeutics (2015) 12:477-490 https://doi.org/10.1007/s13311-015-0343-0}

C.-H. Yang's affiliation appeared incorrectly on the original publication of this article. It appears correctly here.

Publisher's Note Springer Nature remains neutral with regard to jurisdictional claims in published maps and institutional affiliations.

The online version of the original article can be found at https://doi.org/ 10.1007/s13311-015-0343-0

Eyleen L. K. Goh

eyleen.goh@duke-nus.edu.sg

Chih-Hao Yang

chyang@tmu.edu.tw

1 Programme in Neuroscience and Behavioral Disorders, Duke-NUS Graduate Medical School, Singapore, Singapore

2 Lee Kong Chian School of Medicine, Nanyang Technological University, Singapore, Singapore

3 Department of Pharmacology, School of Medicine, College of Medicine, Taipei Medical University, Taipei 110, Taiwan

4 Department of Forensic Medicine, Key Laboratory of Health Ministry for Forensic Science, Xi' an Jiaotong University School of Medicine, Xi'an, Shaanxi, People's Republic of China
Department of Physiology, Yong Loo Lin School of Medicine, National University of Singapore, Singapore, Singapore

6 Department of Pharmacology, College of Medicine, National Cheng Kung University, Tainan, Taiwan

7 Institute for Cell Engineering, Johns Hopkins University School of Medicine, Baltimore, MD, USA

8 Center for Functional Connectomics, Korea Institute of Science and Technology, Seoul, Republic of Korea

9 Institute of Molecular and Cell Biology, A*STAR, Proteos, Singapore, Singapore

10 KK Research Center, KK Women's and Children's Hospital, Singapore, Singapore 